\title{
The biosolubilization of Shenfu coal by Bacillus amyloliquefaciens and Saccharothrix xinjiangensis
}

\author{
XU Yun-long ${ }^{1, a}$, LIU Xiang-rong ${ }^{2, b}$, ZHANG Yao-wen ${ }^{3, c}$ \\ ${ }^{1,2,3}$ College of Chemistry and Chemical Engineering, Xi'an University of Science and Technology, \\ Xi'an 710054, China \\ a1131537462@qq.com, ${ }^{b}$ liuxiangrongxk@163.com, ${ }^{\mathrm{a}} 759060326 @ q q . c o m$
}

\begin{abstract}
Keywords: biosolubilization, Bacillus amyloliquefaciens, Saccharothrix xinjiangensis, coal.
Abstract. In this study, Shenfu crude coal and nitric acid-pretreated coal were solubilized by Bacillus amyloliquefaciens and Saccharothrix xinjiangensis. Around 7-8 \% crude coal dry weight was solubilized within 15 days. The nitric acid pretreatment of the coal increased process efficiency by around $60 \%$ for Saccharothrix xinjiangensis. Elemental analysis and FTIR spectra of $8 \mathrm{~mol} / \mathrm{L}$ nitric acid pretreated coal and biosolubilization products generated by Bacillus amyloliquefaciens and Saccharothrix xinjiangensis showed significant differences between coal and these products.
\end{abstract}

\section{Introduction}

Coal biosolubilization is a promising technology of converting solid coal to liquid fuel or value added chemicals. Compared with thermo-chemical conversion technologies, such as gasification and pyrolysis, it employs microorganisms to liquefy coal under low temperature and atmospheric pressure conditions $^{[1]}$. To date, coal biosolubilization has been widely studied and reported in the literature ${ }^{[2-4]}$.

But majority of current researches merely take lignite, while are lack of jet coal. In the process of coal liquefaction of microbes, the use of different strains, for different coal sample, the secretion of soluble coal actives are also different. The method of acid pretreatment was used to loosen the interaction between coal macromolecules, to improve the conversion rate of dissolved coal in microorganisms, and to analyze the coal products.

\section{Materials and methods}

All of chemical reagents used in this study were analytical grade and were purchased from Beijing and Tianjin Chemical reagents, unless otherwise stated. All experimental data were repeated three times, unless otherwise indicated.

Coal and microorganism. Coal used in this study was sampled from Shenfu, China. After crush separation, coal particles with the diameter ranging from $0.5-0.25 \mathrm{~nm}$ were collected for the experiments. Pretreated coal samples were prepared by putting raw coal particles into $8 \mathrm{~mol} / \mathrm{L}$ nitric acid with a concentration of $0.25 \mathrm{~g} / \mathrm{mL}$ of nitric acid. The treatment lasted for $48 \mathrm{~h}$ at room temperature followed by washing with distilled water until the filtrates became neutral. Then the pretreated coal particles were dried in an oven at $75^{\circ} \mathrm{C}$ for $48 \mathrm{~h}$, and stored in a desiccator before being used for characterization and biosolubilisation. The ultimate compositions of raw and pretreated coal samples were analyzed as shown in table 1.

Table 1 Results of elemental analysis of the coal and oxidized using $8 \mathrm{~mol} / \mathrm{L}$ nitric acid

\begin{tabular}{|c|c|c|c|c|c|c|c|c|}
\hline \multirow{2}{*}{ Samples } & \multicolumn{5}{|c|}{ Elemental analysis $(\%)$} & \multicolumn{3}{|c|}{ Atomic ratio } \\
\hline & $\mathrm{C}_{\mathrm{ad}}$ & $\mathrm{H}_{\mathrm{ad}}$ & $\mathrm{O}_{\mathrm{ad}}$ & $\mathrm{N}_{\mathrm{ad}}$ & $S_{\text {t.ad }}$ & $\mathrm{H} / \mathrm{C}$ & $\mathrm{O} / \mathrm{C}$ & $\mathrm{N} / \mathrm{O}$ \\
\hline Crude coal & 68.23 & 4.86 & 23.96 & 2.68 & 0.27 & 0.85 & 0.26 & 0.13 \\
\hline Coal pretreated $\mathrm{HNO}_{3}$ & 55.04 & 3.73 & 36.15 & 5.08 & 0 & 0.81 & 0.49 & 0.16 \\
\hline
\end{tabular}

The Bacillus amyloliquefaciens and Saccharothrix xinjiangensis bacterial strains were purchased from the China General Microbiological Culture Collection Center and were able to degrade aromatic hydrocarbons and stored in the incubator of the microbiological laboratory. These strains were 
maintained on Nutrient Broth agar slants (NB:10 g tryptone, $3 \mathrm{~g}$ beef extract, $10 \mathrm{~g} \mathrm{NaCl}$, and $15 \mathrm{~g}$ agar per liter of distilled water) at $4{ }^{\circ} \mathrm{C}$.

Biosolubilization of coal. To quantify coal biosolubilization, Bacillus amyloliquefaciens and Saccharothrix xinjiangensis were activated on an NB agar slant at $30^{\circ} \mathrm{C}$ for $24 \mathrm{~h}$ and then incubated in liquid NB medium for $48 \mathrm{~h}$. To obtain experimental cultures, activated Bacillus amyloliquefaciens and Saccharothrix xinjiangensis were added to fresh liquid NB medium (50 $\mathrm{ml}$ in 150-ml flasks). The experimental cultures were then incubated at $30^{\circ} \mathrm{C}$ on a rotary shaker at $150 \mathrm{rpm}$ for $48 \mathrm{~h}$. Sterilized coal was added to the experimental cultures in $1 \%(\mathrm{w} / \mathrm{v})$ proportions and cultured for 15 days. Controls contained only NB medium supplemented with coal (they were not inoculated with bacteria). The residues produced by coal biosolubilization at 3, 6, 9, 12 and 15 days were obtained by centrifuging the cultures at $8,000 \mathrm{rpm}$ for $15 \mathrm{~min}$, washing the residue 5 times with distilled water and drying to a constant weight at $75^{\circ} \mathrm{C}$. During the process of coal biosolubilization, culture aliquots were collected each day under aseptic conditions, centrifuged at 8,000 rpm for $15 \mathrm{~min}$. The A450 of the cell-free supernatants was measured using UV-Vis spectrophotometer to assess coal solubilization .

Table 2 Weight loss rate of crude coal and $\mathrm{HNO}_{3}$ pretreated coal by during its solubilization mediated by Bacillus amyloliquefaciens and Saccharothrix xinjiangensis

\begin{tabular}{lccccc}
\hline \multirow{2}{*}{ strain } & \multicolumn{5}{c}{ weight loss(\%) } \\
\cline { 2 - 6 } & 3rd day & 6th day & 9th day & 12th day & 15th day \\
\hline Bacillus amyloliquefaciens & $5.16 \pm 0.11$ & $6.44 \pm 0.15$ & $6.48 \pm 0.09$ & $6.70 \pm 0.28$ & $8.26 \pm 0.43$ \\
Saccharothrix xinjiangensis & $4.74 \pm 0.21$ & $6.26 \pm 0.23$ & $6.66 \pm 0.42$ & $6.84 \pm 0.32$ & $7.58 \pm 0.75$ \\
control & $1.22 \pm 0.08$ & $1.36 \pm 0.42$ & $2.28 \pm 0.10$ & $2.31 \pm 0.23$ & $2.35 \pm 0.41$ \\
Bacillus amyloliquefaciens $*^{*}$ & $48.81 \pm 0.42$ & $55.10 \pm 0.21$ & $55.40 \pm 0.35$ & $58.48 \pm 0.28$ & $58.94 \pm 0.45$ \\
Saccharothrix xinjiangensis* & $51.78 \pm 0.36$ & $55.43 \pm 0.51$ & $55.93 \pm 0.43$ & $56.25 \pm 0.44$ & $60.52 \pm 0.52$ \\
Control* $^{*}$ & $3.32 \pm 0.15$ & $3.64 \pm 0.04$ & $3.90 \pm 0.18$ & $4.18 \pm 0.16$ & $5.00 \pm 0.14$ \\
\hline
\end{tabular}

$* \mathrm{HNO}_{3}$ pretreated coal

\section{Experimental analysis instrument}

Scanning electron microscope (SEM). Morphology analysis of raw coal (sample A), $\mathrm{HNO}_{3}$ pretreated coal samples (samples B) and residual coal (samples C) were carried out with scanning electron microscope. Low vacuum $(0.075 \mathrm{~mm} \mathrm{Hg})$, low accelerating voltage of $5 \mathrm{kV}$ and magnification of $5000 \times$ were used. The samples were sputter-coated with a layer of gold before SEM analysis the result is Fig. 1.

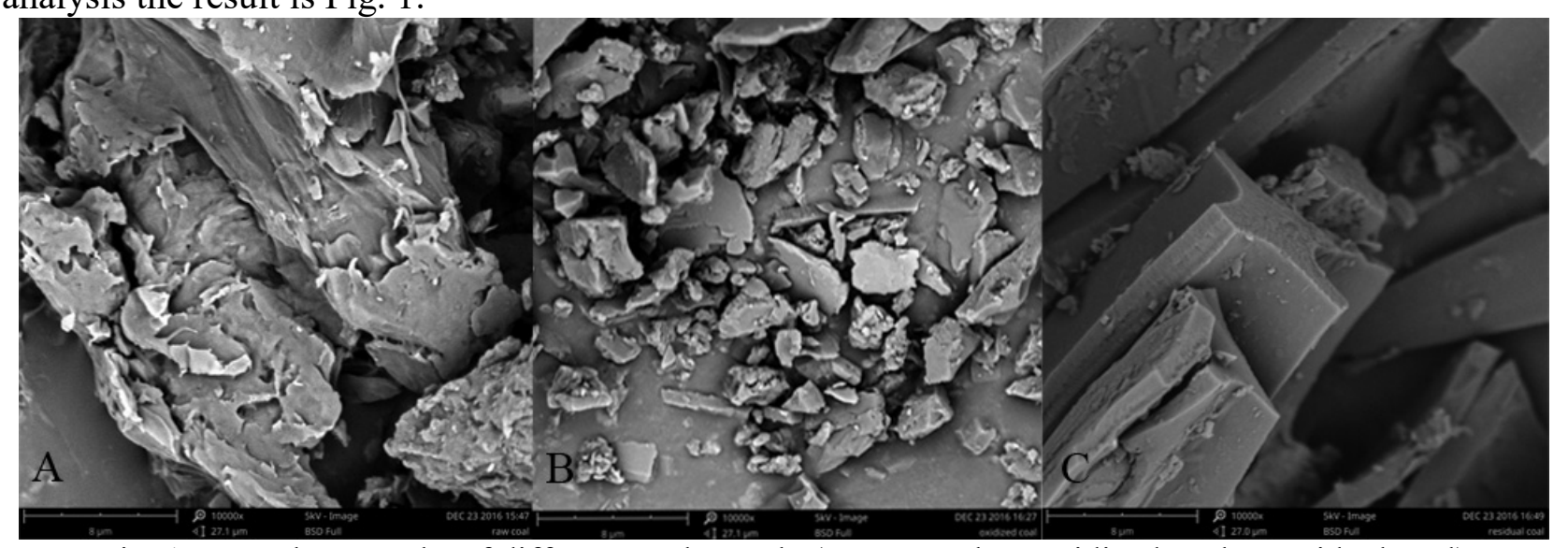

Fig. 1 SEM photographs of different coal sample (A-raw coal; B-oxidized coal C-residual coal)

Fourier transform infrared spectroscopy (FTIR). Spectra were performed by using a transform infrared spectrometer with spectral range of $400-4000 \mathrm{~cm}^{-1}$, FTIR spectra were recorded with a 4 $\mathrm{cm}^{-1}$ spectral resolution. The spectrum is a result of total scan time of 32 the result is Fig. 2. 


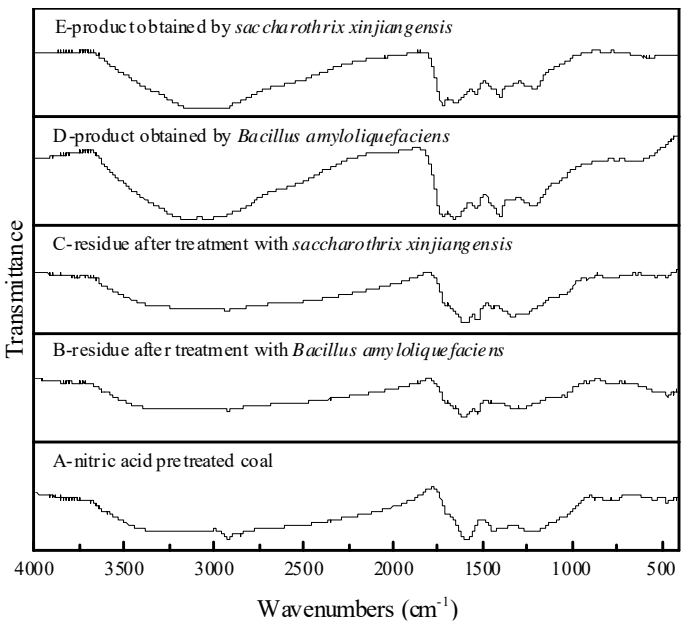

Fig. 2 FTIR spectra of coal and biosolubilisation product

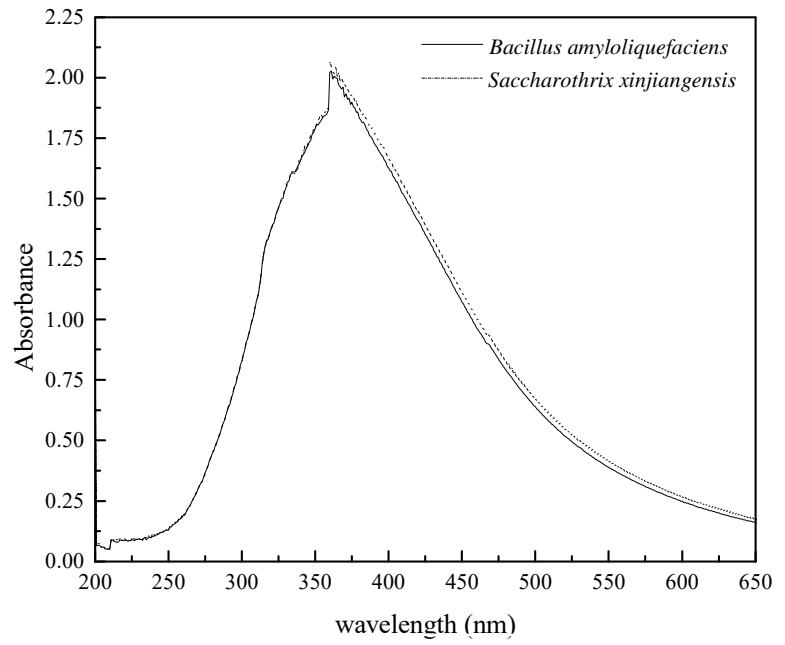

Fig. 3 UV-Vis spectra of biosolubilization coal

UV-Vis spectrum analysis. The product of coal biosolubilization by two kinds of bacteria with water as a reference for spectral scanning ultraviolet spectrum analysis is shown in Fig. 3.

\section{Results and discussion}

Element analysis. Nitric acid pretreatment of coal reduced the contents of carbon, hydrogen and sulfur and increased the contents of oxygen (from 23.96 to $36.18 \%$ ) and nitrogen (from 2.68 to $5.08 \%$ ). Also Kai-yi et al. observed such changes because coal oxidation is based on chemical reactions between nitric acid and functional groups of coal, e.g. carboxylation of aromatic rings, oxidation of side alkyl chains (linked to aromatic rings) to esters, aldehydes and ketones and their nitration $^{[5]}$. These changes in the structure of coal favor its biosolubilization and increase effectiveness of the latter ${ }^{[6-8]}$.

Scanning electron microscope (SEM). The SEM photographs of coal samples with and without pretreatment are shown in Fig. 2. There is small difference of the surface morphology between raw coal (sample A) and $\mathrm{HNO}_{3}$ pretreated coal (sample B). There are more pores in $\mathrm{HNO}_{3}$ pretreated coal (sample B) and surface cracks occurred due to a large number of defects and channels formed in the sample after removal of minerals. The surface morphology of coal was changed significantly after biosolubilization. Biological dissolving treatment, not only made the interlayer spacing to become bigger, but also changed the coal macromolecular structure and made part of the channel open.

Biosolubilization of coal. Results of coal (crude and nitric acid-pretreated) solubilization by Bacillus amyloliquefaciens and Saccharothrix xinjiangensis (shown in Tables 2). The degree of crude coal solubilization by the two strains attained almost $6 \%$ on the sixth day of this process and was maintained at this level till the 12th day. Then it increased to $8.26 \%$ and almost $7.58 \%$ on the 15 th day for Bacillus amyloliquefaciens and Saccharothrix xinjiangensis, respectively. Within the same period of time, the degree of mechanical coal disintegration in the control samples reached $2.35 \%$. According to the literature, the degree of lignite solubilization by the most efficient fungal strains was around $25 \%$ within 4 weeks $^{[9]}$. The biosolubilization of nitric acid-pretreated coal (48.81 and 51.78\% for Bacillus amyloliquefaciens and Saccharothrix xinjiangensis, respectively) occurred much faster than the conversion of crude coal and within the first 3 days was around ten-fold higher. During the subsequent period, biosolubilization continued and reached values of 58.94 and $60.52 \%$ on the last day. However, the weight loss during the last 3 days (between the 12th and 15th day) was rather small.

The higher increase in the degree of biosolubilization of nitric acid-pretreated coal (measured as an increase in absorbance at $450 \mathrm{~nm}$ ) was achieved within the first 3 days. On the 3rd day this was around 34-fold greater compared to that of crude coal, irrespective of the strain (Fig. 4). This observation provides evidence that coal pretreatment with oxidizing agents causes loosening of its structure and gives rise to more efficient solubilization. This is consistent with the results obtained by elemental 
analysis and scanning electron microscope.

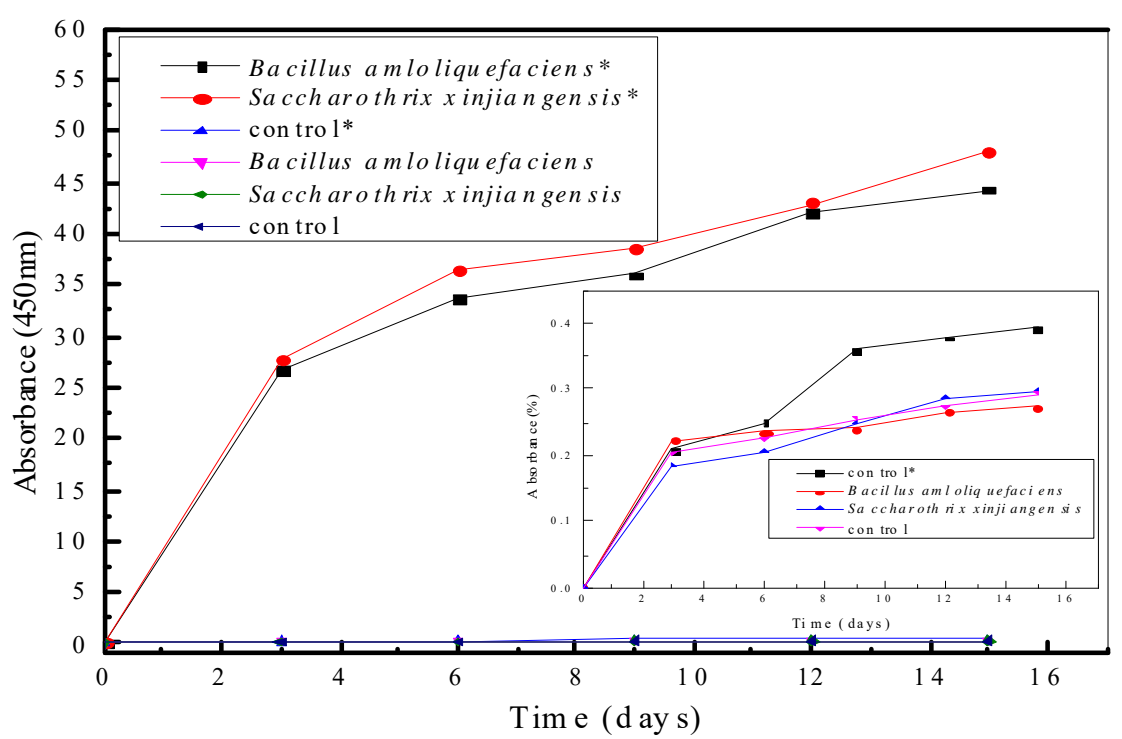

Fig. 4 The biosolubilization of crude and nitric acid-pretreated coal* by Bacillus amyloliquefaciens and Saccharothrix xinjiangensis

FTIR analysis of nitric acid-pretreated coal, products of solubilization and insoluble residues. Comparison of FTIR spectra of nitric acid-pretreated coal and solid residues after its biosolubilization by Bacillus amyloliquefaciens and Saccharothrix xinjiangensis were similar (Fig. 2). Most characteristic is the disappearance of the band at the wavenumber around $1700 \mathrm{~cm}^{-1}$, which is ascribed to carboxylic and carbonyl groups, the narrower absorption band in the range 1300-1000 $\mathrm{cm}^{-1}$ (this band is ascribed to phenol, alcohol and ether groups) in the spectra of solid residues. The band at the wavenumber of $1520 \mathrm{~cm}^{-1}$, which appeared in the consequence of coal oxidation by nitric acid (the presence of group - $\mathrm{NO}_{2}$ ) did not occur in the spectra of residues. The presence of numerous aliphatic- $\mathrm{CH}$ groups in nitric acid-pretreated coal was reflected by the strong band at the wavenumber around $2900 \mathrm{~cm}^{-1}$. The apparent minimum of transmittance at the wavenumber of $1640 \mathrm{~cm}^{-1}$ in the spectra of both product and residues after biosolubilization informed of the presence of aromatic rings as well as $-\mathrm{OH}$ and $-\mathrm{NH}$ groups.

The spectra of biosolubilization products generated by the two bacterial strains were considerably different from the spectrum of nitric acid-pretreated coal (Fig. 2). The bands at 1740 and $1540 \mathrm{~cm}^{-1}$ (ascribed to carboxylic and carbonyl groups as well as $-\mathrm{NO}_{2}$ groups, respectively) were absent in the spectra of liquid products, which contained the new band around $1400 \mathrm{~cm}^{-1}$, which is ascribed to $-\mathrm{COOH}$ groups. The shift of the band characteristic of carbonyl groups towards the smaller wavenumber $\left(1620 \mathrm{~cm}^{-1}\right)$ suggests the occurrence of either conjugated quinones or carboxylic groups connected by hydrogen bonds ${ }^{[10]}$. The spectrum of products generated by Bacillus amyloliquefaciens and Saccharothrix xinjiangensis contained the maximum at $1200 \mathrm{~cm}^{-1}$, which is ascribed to the increased number of alcohol and/or aliphatic ether groups compared to the substrate, supporting the conclusion that coal biosolubilization mechanisms used by the two investigated strains are same. The bands corresponding to linkages $\mathrm{O}-\mathrm{H}$ and/or N-H (in the range of 3500-2900 $\mathrm{cm}^{-1}$ ) were stronger in the spectra of the products. The transmittance in the wavenumber range of $930-660 \mathrm{~cm}^{-1}$ was weaker in the case of the biosolubilization products (irrespective of the strain), which means that they contained less aromatic groups. This finding is consistent with the results of elemental analysis of the nitric acid-pretreated coal and its biosolubilization products.

Ultraviolet-visible spectrum (UV-Vis) of biosolubilisation product. The result of the UV-Vis spectroscopy of the solubilisation product is shown in Fig. 3. The major absorbance in the range of $250-450 \mathrm{~nm}$ indicates that unsatisfied chemical bonds exist in the solubilisation product ${ }^{[11]}$. In particular, absorbance in the $260-300 \mathrm{~nm}$ range indicates that aromatic structures existed in the solubilisation product. The aromatic ring is the main structure unit of coal. Since lots of aromatic ring 
units in coal are connected by complex cross links, coal exists as solid. But after biosolubilisation, aromatic structures were found in liquid, indicating the two bacterial strains could break some cross links in coal so that some simple aromatic rings were released from coal and formed liquid. The above $\mathrm{UV}-\mathrm{Vis}$ results were consistent with the results from infrared spectrum analysis.

\section{Conclusions}

Bacillus amyloliquefaciens and Saccharothrix xinjiangensis, which are capable of degrading aromatic hydrocarbon, were found to solubilize coal. Nitric acid-pretreatment of coal increased biosolubilization efficiency by around $60 \%$. Results of elemental analysis as well as FTIR and UV-Vis spectra confirmed that the mechanisms of biosolubilization of nitric acid-pretreated coal were same. The FTIR and UV-Vis spectra provided evidence that the strains of bacterial caused breaking of certain covalent bonds, occurring in the coal, and the appearance of novel linkages. The results of this study demonstrate that Bacillus amyloliquefaciens and Saccharothrix xinjiangensis were capable of efficient biosolubilization of nitric acid-pretreated coal. The SEM provided evidence that nitric acid-pretreated coal, not only made the interlayer spacing to become bigger, but also changed the coal macromolecular structure and made part of the channel open for. Meanwhile, provide the conditions for coal and bacteria effective contact.

\section{Acknowledgements}

This work was financially supported by the National Natural Science Foundation of China (21373158) and Shaanxi Science and Technology Agency of Sino-Australian Cooperation Projects (2013KW17).

\section{References}

[1] Fakoussa R. M., Frost P. J. In vivo-decolorization of coal-derived humic acids by laccase-excreting fungus Trametes versicolor[J]. Appl., Microbiol. Biotechnol. 1999, 52: 60-65.

[2] Basaran Y., Denizli A., Sakintuna B., et al. Bio-liquefaction of low-rank Turkish lignites and characterization of the products[J]. Energy Fuels, 2003,17: 1068-1074.

[3] Cohen M. S., Bowers W. C., Aronson H., et al. Cell-Free Solubilization of Coal by Polyporus versicolor[J]. Appl. Environ. Microbiol., 1987 52: 2840-2843.

[4] Elbeyli L. Y., Palantoken A., Pikin S., et al. Liquefaction/solubilization of low-rank Turkish coals by white-rot fungus (Phanerochaete chrysosporium)[J]. Energy Sources, 2006, 28: 1063-1073.

[5] SHI Kai-yi, TAO Xiu-xiang, HONG Fen-fen, et al., Mechanism of oxidation of low rank coal by nitric acid[J], Journal of Coal Science and Engineering, 2012, 18(4): 396-399.

[6] SHI Kai-yi, TAO Xiu-xiang, YIN Su-dong, et al., Bio-liquefaction of Fushun lignite: characterization of newly isolated lignite liquefying fungus and liquefaction products[J], Procedia Earth and Planetary Science, 2009, 1: 627-633.

[7] TAO Xiu-xiang, PAN Lan-ying, SHI Kai-yi, et al., Bio-solubilization of Chinese lignite I: extra-cellular protein analysis[J], Mining Science and Technology, 2009, 19: 0358-0362.

[8] YIN Su-dong, TAO Xiu-xiang, SHI Kai-yi, et al., Biosolubilisation of Chinese lignite[J], Energy, 2009, (34): 775-781.

[9] JIANG Feng, LI Zhao-hu, LV Zhi-wei, T. Gao, et al., The biosolubilization of lignite by Bacillus sp. Y7 and characterization of the soluble products[J], Fuel, 2013, (103): 639-664.

[10]HELENA M., KAMILA P., ANNA P., Microbial degradation of low rank coals[J], Fuel, Processing Technology, 2002, (77-78): 17-23.

[11]SHI Kai-yi, YIN Su-dong, TAO Xiu-xiang, et al., Quantitative measurement of coal bio-solubilization by ultraviolet-visible spectroscopy[J]. Energy sources, 2013, (35): 1456-1462. 